\title{
Alteration of Synaptic Network Dynamics by the Intellectual Disability Protein PAK3
}

\author{
Aline Dubos, ${ }^{1}$ Gaelle Combeau, ${ }^{3}$ Yann Bernardinelli, ${ }^{1}$ Jean-Vianney Barnier, ${ }^{3}$ Oliver Hartley, ${ }^{2}$ Hubert Gaertner, ${ }^{2}$ \\ Bernadett Boda, ${ }^{1 *}$ and Dominique Muller ${ }^{1 *}$ \\ Departments of ${ }^{1}$ Basic Neurosciences and ${ }^{2}$ Structural Biology and Bioinformatics, School of Medicine, University of Geneva, 1211 Geneva 4, Switzerland, \\ and ${ }^{3}$ Centre de Neurosciences Paris Sud, Unité Mixte de Recherche 8195, Centre National de la Recherche Scientifique-Université Paris Sud, 91405 Orsay, \\ France
}

Several gene mutations linked to intellectual disability in humans code for synaptic molecules implicated in small GTPase signaling. This is the case of the Rac/Cdc42 effector p21-activated kinase 3 (PAK3). The mechanisms responsible for the intellectual defects and the consequences of the mutation on the development and wiring of brain networks remain unknown. Here we show that expression of PAK3 mutants, suppression of PAK3, or inhibition of PAK3 function in rat hippocampal slice cultures interfere with activity-mediated spine dynamics. Inhibition of PAK3 resulted in two main alterations: (1) an increased growth of new, unstable spines, occurring in clusters, and mediated by activity; and (2) an impairment of plasticity-mediated spine stabilization interfering with the formation of persistent spines. Additionally, we find that PAK3 is specifically recruited by activity from dendrites into spines, providing a new mechanism through which PAK3 could participate in the control of both spine stabilization and local spine growth. Together, these data identify a novel function of PAK3 in regulating activity-mediated rearrangement of synaptic connectivity associated with learning and suggest that defects in spine formation and refinement during development could account for intellectual disability.

\section{Introduction}

p21-activated kinase 3 (PAK3) is one of many genes for which mutations have been implicated in the pathogenesis of intellectual disability (Ropers, 2008). Five mutations have been identified so far, resulting predominantly in a cognitive impairment (Allen et al., 1998). PAK3 belongs to a family of serine/threonine kinases known to participate in the control of actin cytoskeleton dynamics and is believed to act downstream of Rac/Cdc42 GTPases. Previous work has shown that expression of mutated forms of $P A K 3$ or downregulation of PAK3 lead to morphological spine abnormalities, including an increased proportion of thin, immature spines (Boda et al., 2004; Kreis et al., 2007), and variable defects in synaptic plasticity (Boda et al., 2004; Meng et al., 2005; Rex et al., 2009). PAK3 has thus been proposed to participate in the regulation of spine size and morphology as well as several other molecules implicated in Rho GTPase signaling, such as kalirin-7, ARHGEF6, DISC-1, or Cdc42 (Xie et al., 2007;

\footnotetext{
Received June 26, 2011; revised Nov. 9, 2011; accepted Nov. 11, 2011

Author contributions: A.D., B.B., and D.M. designed research; A.D., Y.B., B.B., and D.M. performed research; G.C., J.-V.B., O.H., and H.G. contributed unpublished reagents/analytic tools; A.D., Y.B., B.B., and D.M. analyzed data; A.D., B.B., and D.M. wrote the paper.

${ }^{*}$ B.B. and D.M. contributed equally to this work.

The authors declare no competing financial interests.

This work was supported by the Lejeune Foundation (B.B.) Swiss National Science Foundation Grant 31003A10571, and National Centres for Competence in Research Synapsy (D.M.). We thank Manuel Mameli for advice and guidance in the MNI uncaging experiments, C. Luscher for giving access to the uncaging setup, Z. Jia for providing Pak3 K0 mice, and L. Jourdain for excellent technical skills.

Correspondence should be addressed to Prof. Dominique Muller, Department of Basic Neurosciences, School of Medicine, University of Geneva, 1211 Geneva 4, Switzerland. E-mail: Dominique.Muller@unige.ch.

DOI:10.1523/JNEUROSCI.3252-11.2012

Copyright $@ 2012$ the authors $\quad 0270-6474 / 12 / 320519-09 \$ 15.00 / 0$
}

Hayashi-Takagi et al., 2010; Murakoshi et al., 2011; Penzes et al., 2011).

The mechanisms through which these alterations lead to intellectual disability remain, however, unclear. Notably, an open question is whether mutation of $P A K 3$ could perturb the wiring of developing cortical networks. Evidence from both in vitro and in vivo analyses has shown that excitatory synapses can be formed and eliminated throughout life (Holtmaat and Svoboda, 2009). This phenomenon is particularly important during critical periods of development when experience and activity play a central role in shaping the growth and specificity of synaptic connections. This is achieved through an activity-dependent formation of new synaptic contacts and a selective stabilization of synapses, allowing in this way a fine tuning of synaptic networks (De Roo et al., 2008). This process has been shown recently to play a critical role in different aspects of behavioral learning (Xu et al., 2009; Yang et al., 2009; Wilbrecht et al., 2010; Bednarek and Caroni, 2011). We therefore tested here whether inhibition of PAK3 could interfere with the mechanisms through which activity regulates adaptation of synaptic networks.

\section{Materials and Methods}

Organotypic slices cultures and transfection. Transverse hippocampal organotypic slices cultures were prepared from neonate rats or mice as described previously (Stoppini et al., 1991) using a protocol approved by the Geneva Veterinarian Office (authorization 31.1.1007/3520/0) and maintained for $15-18 \mathrm{~d}$ in culture. Slice cultures were transfected with plasmids encoding fluorescent proteins (pcDNA3.1-EGFP, pCXmRFP1; gift from A. K. Hadjantonakis and R. Y. Tsien, University of California, San Diego, La Jolla, CA) for the control condition or fluorescent proteins together with the dominant-negative PAK3 mutant 
(pcDNA3.1-PAK3-R419X) using a biolistic method (Helios Gene Gun; Bio-Rad). Transfections were performed in slice cultures at $7-8 \mathrm{~d}$ in vitro (DIV), and the effects of expression of PAK3-R419X analyzed 3-4 d later. Plasmid DNAs at a ratio of $7.5 \mu \mathrm{g}$ of pcDNA3.1-EGFP or pCXmRFP1 to $15 \mu \mathrm{g}$ of pcDNA3.1-PAK3 mutants were precipitated onto 5 $\mathrm{mg}$ of $1.6 \mu \mathrm{m}$ gold microcarriers. Fluorescence started to be expressed after $24-48 \mathrm{~h}$ and remained stable for at least $15 \mathrm{~d}$. Control experiments for cotransfection showed that cells cotransfected with pCX-mRFP1 and EGFP-C1-PAK3 (1:2 ratio) expressed both green and red fluorescence in almost all cases. Previous work also showed that overexpression of WT PAK3 did not affect spine shapes or density (Boda et al., 2004).

Peptide and treatments. A peptide from the HIV protein TAT, described for its ability to penetrate into cells, was fused to the rat PAK3 autoinhibitory domain (corresponding to PAK3 amino acids 78-146) to produce a cell-permeating dominant-negative TAT inhibitory peptide (TAT-IP), YGRKKRRQRRRHTIHVGFDAVTGEFTGIPEQWARLLQT SNITKLEQKKNPQAVLDVLKFYDSKETVNNQKYMSFTSGDKS. The length of TAT-IP ( 80 residues) necessitated synthesis via two fragments (1-36 and 37-80), which were assembled by native chemical ligation (Dawson et al., 1994). Because this approach requires $\mathrm{N}$-terminal Cys on the C-terminal fragment, it was necessary to replace $\mathrm{Gln}^{37}$ in the TAT-IP sequence by a Cys residue. This residue was alkylated with iodoacetamide after the ligation step to yield a $\mathrm{Gln}$ analog (side chain $\mathrm{CH}_{2}-\mathrm{S}_{-} \mathrm{CH}_{2}-$ $\mathrm{CONH}_{2}$ rather than $\mathrm{CH}_{2}-\mathrm{CH}_{2}-\mathrm{CONH}_{2}$ ). TAT (YGRKKRRQRRRKG) and TAT-IP were N-terminal labeled with 6-carboxyfluorescein. Authenticity of the synthetic products was verified by mass spectrometry, with purity $>95 \%$ as determined by analytical HPLC. The TAT peptides were dissolved in DMEM two times and used at 1-20 $\mu \mathrm{M}$. A time course analysis of peptide incorporation in slices showed a progressive increase over 3-5 h, followed by a decrease over the next $24-48$ h. IPA-3 (SigmaAldrich) was used at $15 \mu \mathrm{M}$, and NBQX (Tocris Bioscience) and D-APV (Nascent) at 10 and $50 \mu \mathrm{M}$, respectively.

In vitro kinase assay. For transfection, HeLa cells were incubated for $4 \mathrm{~h}$ with $1 \mu \mathrm{g}$ of HA-PAK3a-wt encoding plasmid with or without $0.3 \mu \mathrm{g}$ of GFP-Cdc42-V12 encoding plasmid using Lipofectamine LTX Plus Reagent (Invitrogen). Twenty-two hours after transfection, cells were incubated with $5 \mu \mathrm{M}$ TAT or TAT-IP peptide. Two hours later, cells were lysed, and the phosphorylation assays were performed as described (Kreis et al., 2007). Quantification of PAK3 inhibition was achieved by analyzing the levels of myelin-based protein (MBP) phosphorylation, normalized to values of immunoprecipitated HA-PAK3.

Confocal imaging and image analysis. Laser-scanning microscopy was realized with an Olympus Fluoview 300 system using a $488 \mathrm{~nm}$ argon laser and a $537 \mathrm{~nm}$ helium-neon laser. Repetitive confocal imaging experiments were performed as described (De Roo et al., 2008). The analyses of spine turnover and stability were performed blind on individual $z$-stacks of raw images as described (De Roo et al., 2008). Analyses were made on dendritic segments of $\sim 35-40 \mu \mathrm{m}$ in length and located between 100 and $200 \mu \mathrm{m}$ from the soma on secondary or tertiary dendrites in the middle third of the stratum radiatum, with spine densities varying between 0.8 and 1.2 spines $/ \mu \mathrm{m}$. The same segments were imaged repetitively over several days, and each protrusion was identified on individual $z$-stacks using a plugin made for the OsiriX software. A total of 1765 protrusions, including 488 newly formed ones, were analyzed under control conditions (12 EGFP- and 5 mRFP-transfected cells) and compared with 1599 protrusions and 538 newly formed ones after PAK3 inhibition (12 R419X-PAK3-transfected cells, 4 TAT-IP, and 5 IPA-3-treated cells). We counted as new protrusions all new structures (spines or filopodia) appearing between two observations ( 5 or $24 \mathrm{~h}$ ) and characterized by a length of $>0.4 \mu \mathrm{m}$. For disappearances, we counted all protrusions (spines and filopodia) that could no longer be identified on the next observation. Protrusions without enlargement at the tip were identified separately as filopodia, but because they represented only a small fraction of total protrusions found at the beginning of the experiment $(0.9$ and $1.7 \%$ for control and PAK3 inhibition conditions) and of newly formed protrusions ( 4.3 and $6.3 \%$, respectively), we included them in the analyses of spine turnover. Dubious situations attributable to possible changes in protrusion shape, size, or orientation were discarded but overall accounted for only a small number of cases $(<2 \%)$. Images in figures are maximum intensity projections of $z$-stacks treated with the volume rendering algorithm of OsiriX software.

To analyze the clustered growth of protrusions after inhibition of PAK3, we plotted for all dendritic segments the location of all new spines formed over a period of $24 \mathrm{~h}$ and measured the interspine distances. To compare conditions with different rates of spine growth and thus different interspine distances, we analyzed two parameters: (1) the symmetry or skewness of the interspine interval distributions calculated for each dendritic segment using the algorithm provided by GraphPad Prism software; and (2) the fraction of interspine intervals shorter than the mean interspine interval that somehow compares the mean and the median of the distribution. The mean interspine intervals in these experiments were $3.0 \pm 0.3 \mu \mathrm{m}$ for EGFP and $3.1 \pm 0.4 \mu \mathrm{m}$ for TAT versus $2.3 \pm 0.3 \mu \mathrm{m}$ for R419X-PAK3 and $2.2 \pm 0.3 \mu \mathrm{m}$ for TAT-IP conditions.

Glutamate uncaging experiments. Slices were transfected with pDsRedExpress together with EGFP-C1 vector (Clontech) fused with the wildtype mouse PAK3, pcDNA3.1-EGFP-PAK3. Imaging and glutamate uncaging were performed using an Olympus FluoView 300 system with a two-photon laser (Mai Tai HP; Spectra-Physics) and 40× immersion objective. Beam intensity and pulse duration were controlled by an electro-optical modulator (Pockels cells, EOM; Conoptics). DsRedExpress was visualized at $916 \mathrm{~nm}$ and EGFP-PAK3 at $780 \mathrm{~nm}$, and uncaging was performed at $725 \mathrm{~nm}$. 4-Methoxy-7-nitroindolinyl-glutamate (MNI-Glu; $3 \mathrm{~mm}$; Tocris Bioscience) was applied in the culture medium. Glutamate uncaging was achieved using repetitive 1.5 ms pulses $(1 \mathrm{~Hz}$, three to eight pulses, laser power of $70 \mathrm{~mW}$ ). Whole-cell patch-clamp recordings confirmed that these light pulses applied close to spines on secondary dendrites of CA1 pyramidal neurons reproducibly evoked synaptic responses (see Fig. 5A; $n=5$; Mameli et al., 2011). The amount of photo-released glutamate was adjusted by changing the number of pulses. However, because the analyses of PAK3-EGFP distribution could not be performed under patch-clamp conditions, we used as additional criteria of successful synaptic activation the enlargement of the spine head produced by the uncaging protocol and analyzed the variations of PAK3 distribution in those cases. No changes in PAK3 distribution occurred in spines in which the uncaging did not result in enlargement. Image analyses were performed with NIH Image J software by calculating the sum of pixel intensities on fixed regions of interest covering complete stacks of individual spines or neighboring dendritic segments. To compare images taken at different times, with different wavelengths and from different preparations, we calculated ratios of green/red fluorescence in the spine head, the adjacent dendrite, and a distal part of the dendrite (>3 $\mu \mathrm{m}$ apart), and we normalized in each experiment the values obtained in the spine head with those in the adjacent dendrite or those in the adjacent dendrite with those in the distal dendrite ( $>3 \mu \mathrm{m}$ apart). These values were then compared before and 10-15 min after uncaging. The changes in volume of spine heads were estimated by comparing the sum of pixel intensities of DsRedExpress fluorescence of identified spines on $2 \mathrm{D}$ projection of stacked images.

Stimulation and calcium imaging experiments. Slice cultures were maintained at $32^{\circ} \mathrm{C}$ in an interface chamber, and EPSPs were evoked by stimulation of a group of Schaffer collaterals. LTP was induced by theta burst stimulation (TBS) (five trains at $5 \mathrm{~Hz}$ composed each of four pulses at $100 \mathrm{~Hz}$, repeated twice at $10 \mathrm{~s}$ intervals). For calcium imaging, mRFPexpressing cells were loaded with the cell-permeable calcium indicator Fluo-4 AM (F-14201; Invitrogen) as described previously (De Roo et al., 2008). Calcium transients evoked by three to six consecutive stimulations were recorded in 10-25 spines per dendritic segment using line scans through spine heads. Spines were considered as activated whenever the Fluo-4 AM fluorescence signal $\left(\Delta F / F_{0}\right)$ increased by $>20 \%$ over background. LTP was induced by TBS using the same stimulation pulses. The dendritic segments were then reimaged over the next days. Spines were considered to enlarge whenever the diameter of the spine head had increased by $0.1 \mu \mathrm{m}$ between before and $5 \mathrm{~h}$ after TBS.

Statistics. All data are given as mean with the SEM. Unless mentioned, statistical analyses were performed using standard $t$ tests. Mann-Whitney $U$ tests were used when a normality test failed. $\alpha$ was set to $5 \%$ for all tests. 

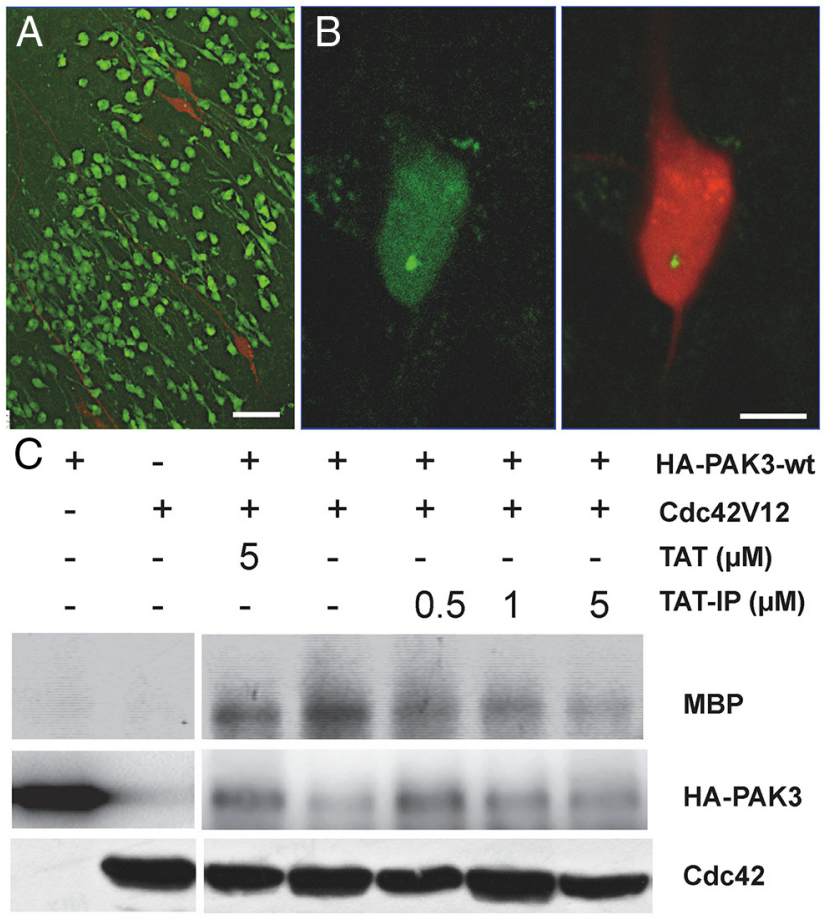

HA-PAK3

Cdc42

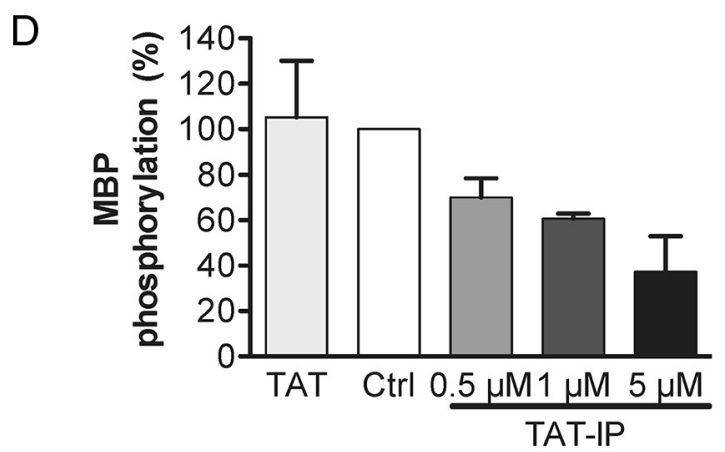

Figure 1. Inhibition of PAK3 activity by TAT-IP. $\boldsymbol{A}$, Illustration of the penetration into CA1 neurons of TAT-IP labeled with fluorescein among which are a few mRFP-transfected cells (scale bar, $50 \mu \mathrm{m}$ ). B, Illustration of an mRFP-transfected cell (right, red) loaded with fluorescein-labeled TAT-IP (left, green; scale bar, $10 \mu \mathrm{m}$ ). C, Autoradiogram showing the concentration dependency of MBP phosphorylation with PAK3 being activated by a constitutively active mutant of Cdc42 (Cdc42-V12). Note the progressive inhibition of MBP phosphorylation by increasing concentrations of TAT-IP. The figure also shows the levels of immunoprecipitated HA-PAK3 and Cdc42. D, Quantitative assessment of MBP phosphorylation normalized to HA-PAK3 levels as a function of different concentrations of TAT-IP $(n=2-5)$.

\section{Results}

Inhibition of PAK3 promotes spine growth

To analyze the role of PAK3 on spine dynamics, we transfected CA1 neurons in hippocampal slice cultures at 7-8 DIV with EGFP or mRFP using a biolistic approach and, 3-4 d later, monitored identified dendritic segments over several consecutive days using confocal microscopy. Several approaches were used to interfere with PAK3 function. First, we expressed a dominantnegative mutant of PAK3 resulting in a truncated protein devoid of kinase activity (Allen et al., 1998). Second, we synthesized a fusion peptide between a TAT sequence and the autoinhibitory domain of PAK3 (amino acids 78-146; TAT-IP). Labeling of these peptides with fluorescein revealed a rapid penetration into neurons within the first $24 \mathrm{~h}$, and assessment of the efficiency of TAT-IP by in vitro kinase assay showed a dose-dependent reduction of MBP phosphorylation (Fig. 1C). With 5-10 $\mu \mathrm{M}$ TAT-IP, MBP phosphorylation was significantly reduced by $63 \%(n=$ $4-5 ; p<0.05, t$ test). As a third approach, we tested the effect of
IPA-3, an allosteric inhibitor of group I PAK. Finally, to further assess specificity, we performed experiments in hippocampal slice cultures prepared from PAK3 knock-out mice (Meng et al., 2005).

All these approaches consistently showed that inhibition of PAK3 affected the mechanisms of spine growth. We assessed turnover in these experiments by measuring all new protrusions formed and lost over consecutive periods of $24 \mathrm{~h}$. Filopodia, defined as protrusions without enlargement at the tip, were included in these counts because they represented only a small fraction of all newly formed protrusions $(4.3 \%$ or 21 of 488 newly formed protrusions analyzed under control conditions (EGFP- or mRFP-transfected cells) versus $6.3 \%$ or 34 of 538 new protrusions analyzed after PAK3 inhibition; $p=0.21$, Fisher's exact test). Thus, although the proportion of filopodia tended to increase after inhibition of PAK3, spines represented the vast majority of newly formed protrusions under all conditions analyzed. The average head diameter of these newly formed spines observed under control conditions or after inhibition of PAK3 did not differ $(0.46 \pm 0.01 \mu \mathrm{m}$ for EGFP and mRFP vs $0.47 \pm 0.01 \mu \mathrm{m}$ for PAK3 inhibition; 488 and 538 new spines analyzed). However, newly formed spines observed after inhibition of PAK3 tended to be longer $(1.21 \pm 0.06 \mu \mathrm{m}$ for PAK3 inhibition vs $1.03 \pm 0.03 \mu \mathrm{m}$ for EGFP and mRFP; $p<0.05$, Mann-Whitney $U$ test), consistent with previous analyses of spine morphology (Boda et al., 2004).

Expression for 3-4 d of the R419XPAK3 mutant in CA1 pyramidal neurons resulted in a marked increase in the rate of new spine formation per $24 \mathrm{~h}$, assessed over 2 consecutive days in 12 EGFP control neurons and 12 R419X-PAK3 and EGFP cotransfected cells (Fig. 2E; EGFP: $18.0 \pm$ $1.5 \%, 12$ cells, 171 new spines analyzed, 24 observation periods vs R419X-PAK3: $28.8 \pm 2 \%$ of new spines per $24 \mathrm{~h}, 12$ cells, 231 newly formed spines analyzed, 24 observation periods; $p<0.001, t$ test). This effect was limited to growth and did not affect spine loss (Fig. $2 F ; n=24, p>0.5)$ and thus resulted in an increase in spine density (Fig. $2 G ; n=36, p<0.05$, ttest). Similarly, treatment of slice cultures for $24 \mathrm{~h}$ with $5 \mu \mathrm{M}$ TAT-IP or $15 \mu \mathrm{M}$ IPA-3 resulted in a similar increase in the rate of spine growth (TAT-IP: $35.0 \pm 1.8 \%$ of new spines per $24 \mathrm{~h}, 4$ cells, 84 new spines analyzed, 4 observation periods vs TAT: $23.0 \pm 2.4 \%$ of new spines, 5 cells, 56 new spines analyzed, 7 observation periods; $p<0.05$, Mann-Whitney test; IPA-3: $34.2 \pm$ $4.5 \%, 5$ cells, 110 new spines analyzed, 5 periods of analysis vs mRFP: $19.9 \pm 2.2 \%, 6$ cells, 69 new spines analyzed, 12 observation periods; $p<0.01$, Mann-Whitney test; Fig. $2 E$ ). This effect was also selective: the rate of spine disappearance did not change (Fig. $2 F$ ) and the spine density tended to increase (Fig. 2G; IPA3: $p<0.01$, MannWhitney test). These observations were further confirmed by analyzes of slice cultures prepared from Pak3 knock-out or wild-type 
EGFP

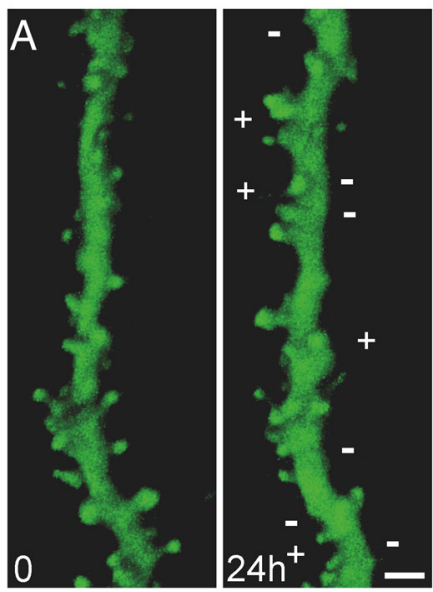

Pak3-WT

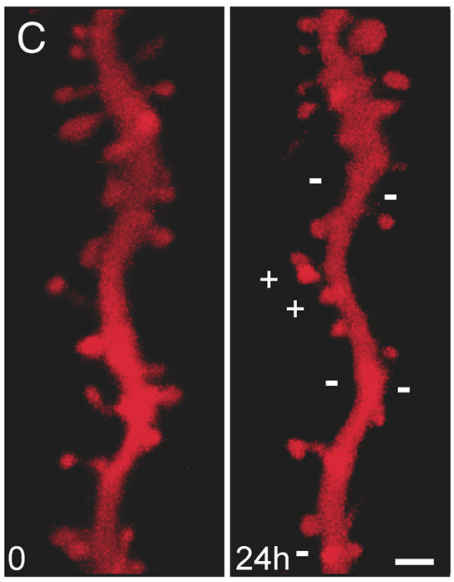

R419X

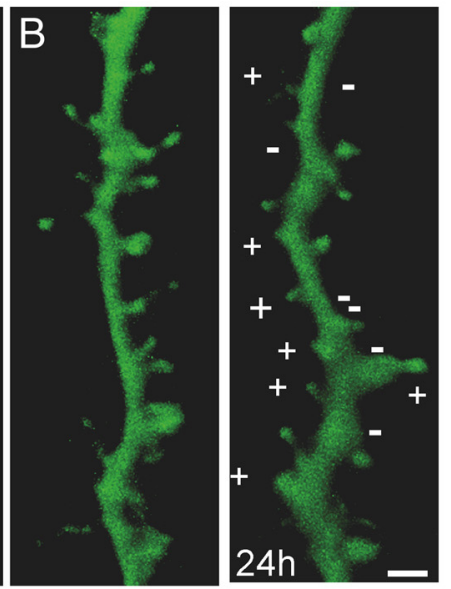

Pak3-KO
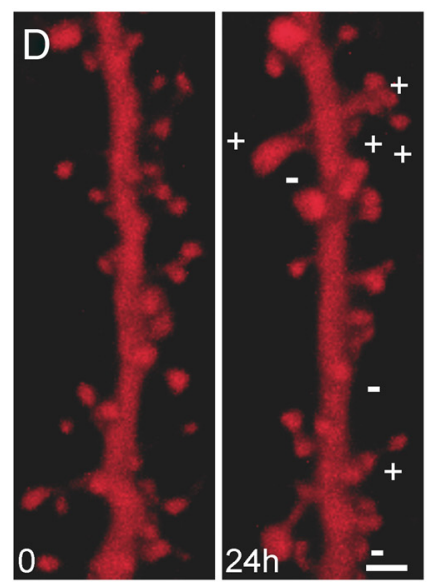
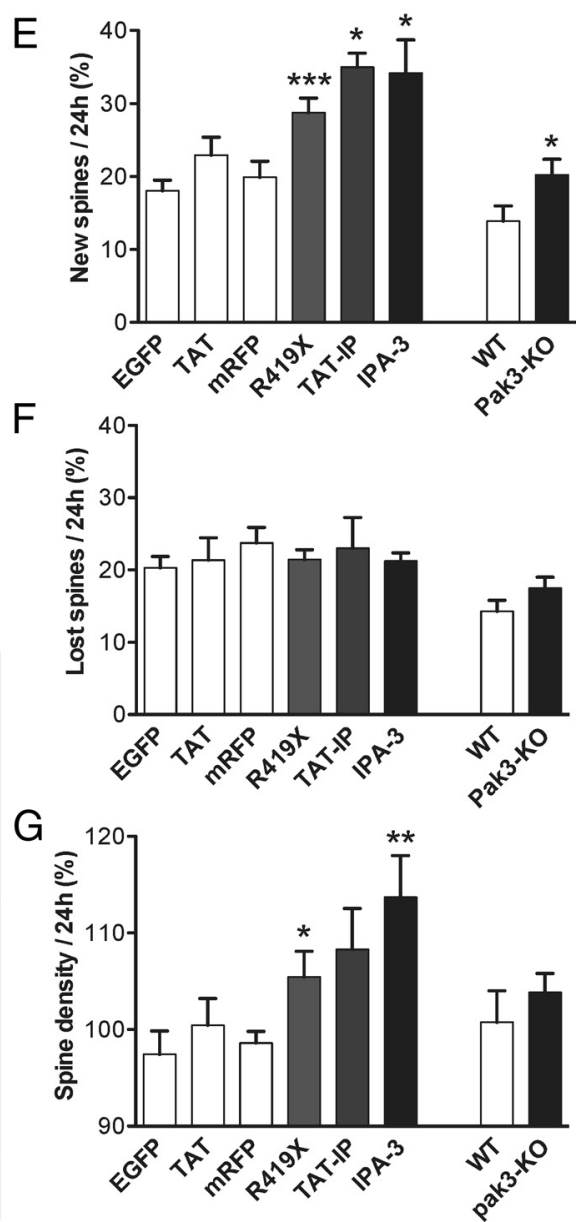

Figure 2. Inhibition of PAK3 function promotesspine growth. $A, B$, Dendriticsegment of CA1 pyramidal neurons imaged at 24 hinterval in an EGFP $(A)$ and R419X-PAK3 + EGFP $(B)$ transfected cell. The plus and minus signsindicatethechanges innewandlostspines (scalebar, $2 \mu \mathrm{m}) . \boldsymbol{C}, \boldsymbol{D}$, mRFP-transfected dendriticsegments ofCA1 neuronsfromWT(C)andPAK3-KOmice(D) illustratedat24hintervals.E, Proportion ofnewspines observed over periods of $24 \mathrm{~h}$ and expressed as percentage of the number of spines present at the first observation under the indicated conditions [EGFP, $n=24 ;$ TAT peptide, $5 \mu \mathrm{m}[\mathrm{scAP}](n=7) ; \mathrm{mRFP}, n=12 ; \mathrm{R} 419 \mathrm{X}-\mathrm{PAK} 3+$ EGFP-transfected cells, $n=24(p<0.001, t$ test); TAT-IP, $n=4(5 \mu \mathrm{m} ; p<0.5$, Mann-Whitney Utest); and IPA-3, $n=5(15 \mu \mathrm{m} ; p<0.05$, Mann-Whitney Utest). The right panel shows the same values obtained in mRFP-transfected cellsfromWTandPAK3-K0 mice $(n=16$ and 19; $p<0.05)$.F, Rate ofspinelossper24h periodandexpressedas percentageofspines presentatthefirstobservation in the sameexperiments. Therightpanel shows the same values for WT and PAK3-KO slice cultures. G, Changes in spine density assessed over $24 \mathrm{~h}$ in the same experiments. ${ }^{*} p<0.05 ;{ }^{* *} p<0.01{ }^{* * * *} p<0.001$.

littermate mice. The rate of new spine formation per $24 \mathrm{~h}$ increased (Pak3-KO: $20.2 \pm 2.1,6$ cells, 105 new spines analyzed, 16 observation periods vs WT: $13.9 \pm 2.1 \%$, 7 cells, 88 new spines analyzed, 19 observation periods; $p<0.05$, Mann-Whitney $U$ test), whereas no changes in the rate of spine loss and spine density were detected. The initial values of spine density in all these different conditions were also comparable (EGFP, $0.93 \pm 0.06 \mu \mathrm{m}^{-1}$; R419X-PAK3, $0.95 \pm 0.03 \mu \mathrm{m}^{-1}$; TAT-IP, $1.01 \pm 0.05$ $\mu \mathrm{m}^{-1}$; TAT, $0.97 \pm 0.05 \mu \mathrm{m}^{-1}$; IPA-3, $\left.0.91 \pm 0.09 \mu \mathrm{m}^{-1}\right)$. Overall, these data indicate that a major function of PAK3 in neurons is to negatively regulate the mechanisms of spine growth.

Spine growth mediated by inhibition of PAK3 occurs in clusters and is activity dependent

A striking characteristic of the increased spine growth triggered by inhibition of PAK3 was its occurrence in small regions (hot-
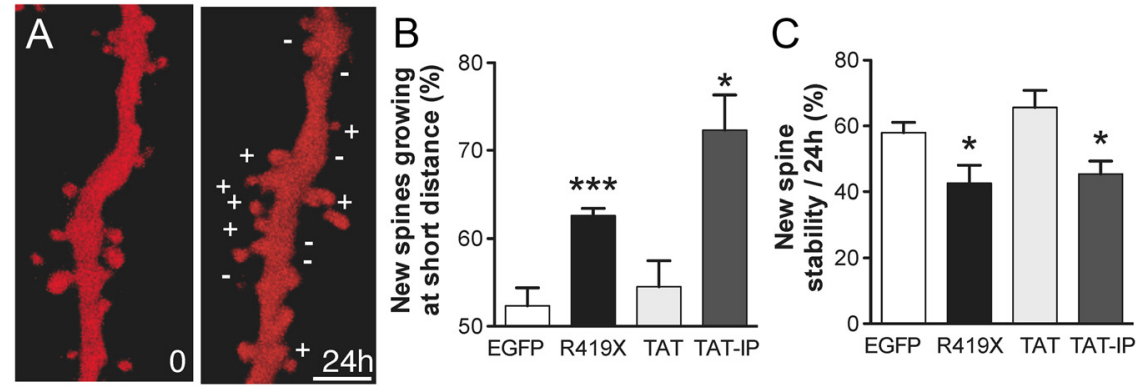

Figure 3. Spinegrowth mediated by PAK3 inhibition occursin clusters. $A$, Spinechanges observed over 24h in a sliceculturetreated with TAT-IP $(5 \mu \mathrm{m})$. Notetheaccumulation of new (+) andlost $(-)$ spines occurringin a small dendritic area (scalebar, $2 \mu \mathrm{m})$. B, Fraction of intervals between new spines detected over $24 \mathrm{~h}$ that are smaller than the mean spine interval under control conditions (EGFP; $n=10)$, R419X-PAK3 (R419X; $n=10 ; p<0.05)$, TAT peptide (TAT; $5 \mu m ; n=4)$, and TAT-IP(TAT-IP; $5 \mu \mathrm{m} ; n=4 ; p<0.001)$. C, Stability at 48 h of new spines formed between 0 and $24 \mathrm{~h}$ under the same conditions (R419X-PAK3; $n=10, p<0.05 ;$ TAT-IP; $n=4, p<0.05$ ). ${ }^{*} p<0.05$; ${ }^{* * *} p<0.001$.

spots) of $2-5 \mu \mathrm{m}$ on a dendritic segment. Figure $3 A$ illustrates this with the same dendritic segment imaged before and $24 \mathrm{~h}$ after application of TAT-IP. Several hotspots could be present on the same dendritic segment and located in a nonspecific manner, regardless of the size or localization of the dendrite or of the spine 
IPA-3

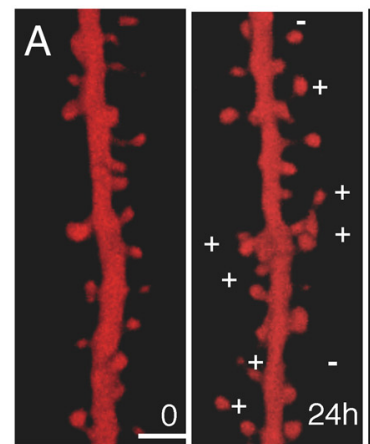

IPA-3 + NBQX/AP5

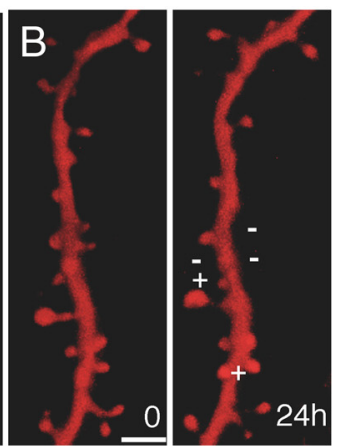

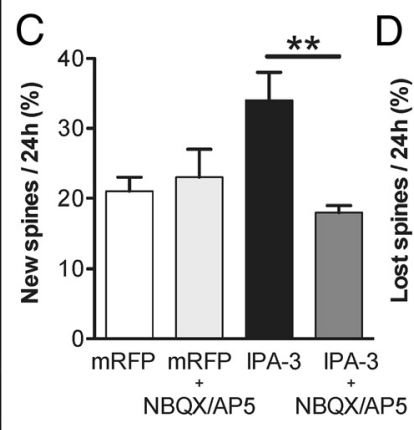

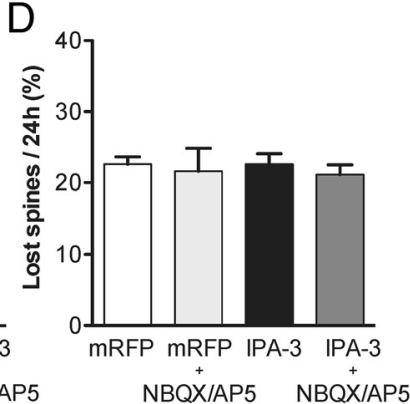

Figure 4. Spine growth mediated by PAK3 inhibition is activity dependent. $\boldsymbol{A}$, Illustration of the $24 \mathrm{~h}$ spine turnover in an mRFP-transfected cell treated with the PAK inhibitor IPA-3 (15 $\mu \mathrm{M})$. $\boldsymbol{B}$, Same but with IPA-3 applied under conditions of synaptic transmission blockade with NBQX (10 $\mu \mathrm{M})+$ AP-5 (50 $\mu \mathrm{M})$. C, Proportion of new spines formed per $24 \mathrm{~h}$ in control, mRFP-transfected cells $(n=5)$, mRFP-transfected cells treated with NBQX and AP-5 $(n=3)$, mRFP-transfected cells treated with IPA-3 $(n=4)$, or IPA-3 together with NBQX and AP-5 ( $n=5, p<0.01)$. D, Proportion of spines lost per $24 \mathrm{~h}$ under the same experimental conditions. ${ }^{* *} p<0.01$.

density. To quantify this phenomenon despite differences in rate of spine formation, we assessed the distance between all new spines formed over a period of $24 \mathrm{~h}$ and analyzed the distribution of the interspine intervals. We reasoned that a clustered growth in small regions should result in a skewed distribution of interspine intervals with an increased proportion of new spines growing at short intervals. Under control conditions (EGFP) or with the TAT peptide (TAT), the distribution of intervals between new spines was quite symmetrical with a skewness averaging $0.6 \pm 0.2$ and $0.8 \pm 0.1$, respectively (EGFP, 10 cells, 120 new spines analyzed; TAT, 5 cells, 76 new spines analyzed). Consistent with this, the proportion of interspine intervals smaller than the mean interspine value represented $52.3 \pm 2.0$ and $54.5 \pm 2.9 \%$, respectively (Fig. $4 B ; n=10$ and 4 cells; mean interspine intervals, $3.0 \pm$ $0.3 \mu \mathrm{m}$ for EGFP and $3.1 \pm 0.4 \mu \mathrm{m}$ for TAT). Thus, spine growth under control conditions occurred in a more or less random manner. In contrast, in cells expressing the R419X-PAK3 mutation or cells treated with the inhibitory peptide (TAT-IP), the skewness of the interspine interval distribution was significantly higher $(1.3 \pm 0.1$ and $2.0 \pm 0.3$, respectively; R419X-PAK3: 12 cells, 231 new spines analyzed; TAT-IP: 4 cells, 84 new spines analyzed; $p<0.05$, Mann-Whitney $U$ test), and a significantly larger fraction of spines grew at intervals shorter than the mean value $(62.6 \pm 0.8$ and $72.3 \pm 4.0 \%, n=11$ and 4 cells; $p<0.05$ and 0.001, Mann-Whitney $U$ test, respectively; mean interspine intervals, $2.3 \pm 0.3 \mu \mathrm{m}$ for R419X-PAK3 and $2.2 \pm 0.3 \mu \mathrm{m}$ for TAT-IP). Accordingly, these results indicate that the growth process triggered by inhibition of PAK3 occurred preferentially at what could represent hotspots.

We then also examined the stability of these new spines by measuring the fraction of new spines detected over a period of $24 \mathrm{~h}$ and still present after $48 \mathrm{~h}$. As shown in Figure $3 C$, the probability that newly formed spines persisted at $48 \mathrm{~h}$ was significantly decreased in R419X-PAK3-expressing cells and in slice cultures treated with TAT-IP (42.6 \pm 5.5 vs $58.0 \pm 3.1 \%$ for R419X-PAK3 and EGFP, $n=10, p<0.05 ; 45.3 \pm 4.0$ vs $65.6 \pm$ $5.1 \%$ for TAT-IP and TAT, $n=5, p<0.05$, Mann-Whitney $U$ test). Thus, spine growth is enhanced, but the stability of these new spines is decreased, a parameter that could account for the variable effects on spine density.

The observation that spines grow in clusters during inhibition of PAK3 suggested a possible role of activity. To assess this possibility, we compared the effect of the PAK inhibitor IPA-3 (15 $\mu \mathrm{M})$ under control conditions and after blockade of excitatory glutamate receptors with NBQX $(10 \mu \mathrm{M})$ and D-AP-5 (50 $\mu \mathrm{M}$; Fig. $4 A, B)$. We combined the two antagonists and used $24 \mathrm{~h}$ applications, conditions that did not affect the basal spine turnover, consistent with the antagonistic effects produced individually by chronic blockade of either AMPA or NMDA receptors (Fig. 4C,D; Lüthi et al., 2001; Mateos et al., 2007). This treatment did not affect spine density (variations in spine density over $24 \mathrm{~h}$ : control, $1.08 \pm 0.1$ to $1.03 \pm 0.09$ spines $/ \mu \mathrm{m}, n=5 ; 205$ spines analyzed; NBQX + D-AP-5, $0.92 \pm 0.05$ to $0.94 \pm 0.05$ spines/ $\mu \mathrm{m}, n=7 ; 247$ spines analyzed). Interestingly, blockade of excitatory synaptic activity completely prevented the growthpromoting effect of IPA-3 (Fig. $4 C ; 34.0 \pm 4.0$ vs $18.0 \pm 1.0 \%$ of new spines per $24 \mathrm{~h}$ for IPA-3 and IPA-3 with NBQX and D-AP-5, respectively, $n=4$ and $5 ; 110$ and 40 new spines analyzed, $p<$ $0.01, t$ test), without affecting spine loss (Fig. $4 D$ ). This result suggested therefore that PAK3 could contribute to the control of spinogenesis by activity.

\section{Activity promotes translocation of PAK3 into dendritic spine heads}

The above results suggested an inhibitory action of PAK3 in dendrites on spine growth, but other studies have also provided evidence for a role of PAK3 in spine heads (Chen et al., 2007). We thus wondered whether activity could recruit PAK3 from dendrites into spines. For this, we used a two-photon glutamate uncaging approach to activate identified dendritic spines and assess the variations in PAK3 localization by expressing an EGFP-PAK3 construct together with DsRedExpress. Glutamate uncaging was achieved using repetitive light pulses (three to eight) of short duration $(1.5 \mathrm{~ms}$ ) at $725 \mathrm{~nm}$. Efficiency of these pulses to release glutamate and evoke synaptic currents was tested electrophysiologically (Fig. 5A; Mameli et al., 2011). Uncaging glutamate under these conditions resulted in an enlargement of the spine head in most spines measured 10-15 min after stimulation (Fig. $5 \mathrm{~B}, \mathrm{C}$ ), a hallmark of plasticity induction (Matsuzaki et al., 2004; Harvey and Svoboda, 2007). In control EGFP-transfected cells, light pulses triggered an enlargement exceeding $15 \%$ in 7 of 10 stimulated spines, with an increase in spine size averaging $38 \pm$ 6\% 10-15 min after stimulation. The same light pulses in the absence of caged glutamate did not affect spine size (size variations, $2 \pm 4 \% ; n=7$; Fig. $5 D$ ). Glutamate uncaging was then applied to cells transfected with DsRedExpress and EGFP-PAK3. Light stimulation triggered an enlargement exceeding $15 \%$ in 11 of 16 stimulated spines averaging $48 \pm 6 \% 10-15$ min after stim- 

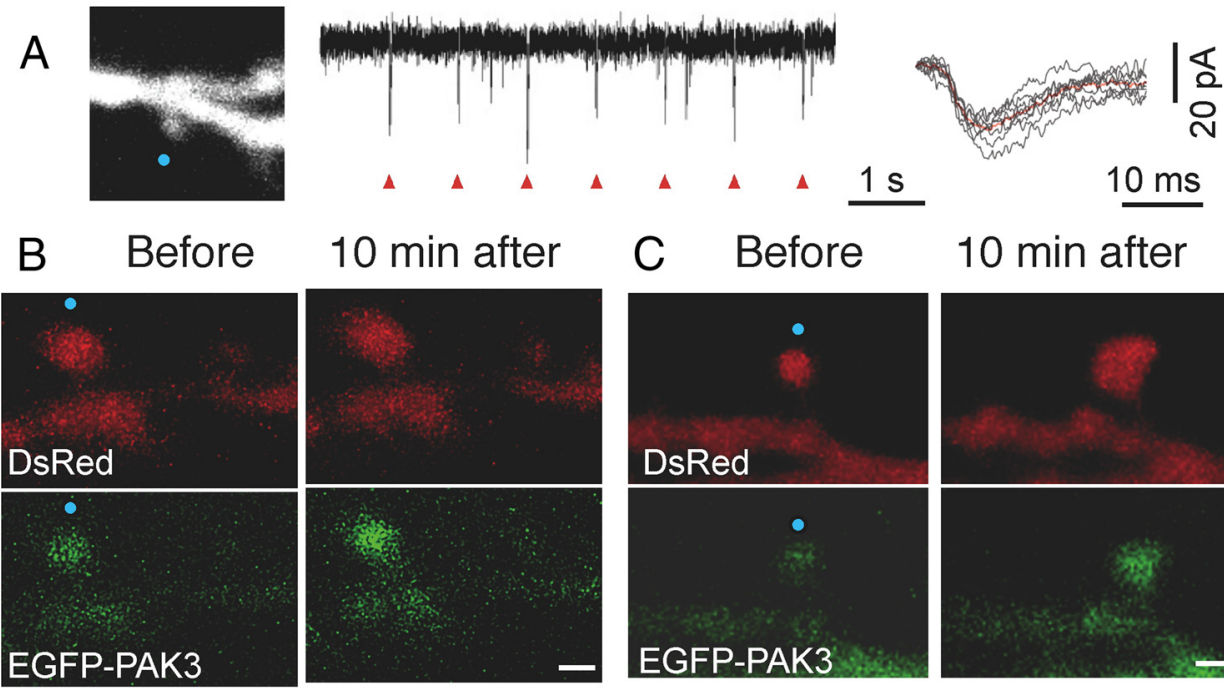

10 min after
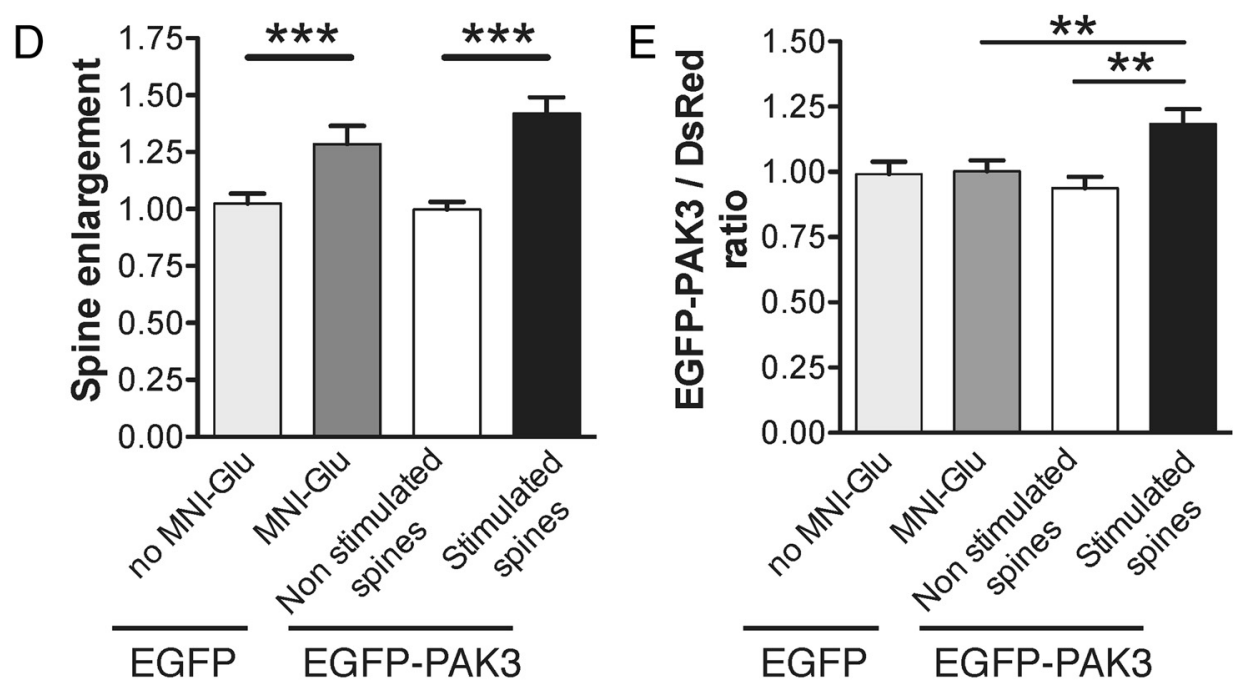

Figure 5. Activation of spines through glutamate uncaging leads to a recruitment of EGFP-PAK3 from dendrite into spines. $A$, Repetitive light stimuli to uncage MNI-Glu in front of a spine (dot) on a secondary dendrite of a CA1 neuron reproducibly evoked currents (red triangles) with characteristics close to synaptic currents (right, red: mean trace). B, C, Dendritic spines of CA1 neurons cotransfected with DsRedExpress and EGFP-PAK3 and imaged before and 10 min after uncaging of MNI-Glu ( $3 \mathrm{~mm}$; dot). Note the enlargement of the spine produced by uncaging (red) and the accumulation of EGFP-PAK3 fluorescence (green) in the light-stimulated spine. $\boldsymbol{D}$, Spine enlargement measured as the sum of pixel intensities before and $10-15$ min after application of light pulses in EGFP-transfected cells (with and without MNI-Glu; $n=7$, $p<0.001)$ and in EGFP-PAK3-transfected cells on stimulated $(n=12)$ and neighboring, nonstimulated spines $(n=10, p<0.001)$. E, Green/red fluorescence ratios measured in spine heads and normalized for each experiment with the values obtained in the adjacent dendrite. Under control conditions (EGFP + DsRedExpress cotransfection), this ratio remained closed to 1 with $(n=7)$ or without $(n=7)$ MNI-Glu uncaging. In contrast, in EGFP-PAK3-transfected cells, the green/red ratio was significantly increased in stimulated spines $(n=12)$ versus nonstimulated spines $(n=10, p<0.01)$, indicating a greater accumulation of EGFP-PAK3 versus DsRedExpress in spine heads. ${ }^{* *} p<0.01 ;{ }^{* * *} p<0.001$.

ulation ( $n=11, p<0.001)$. Nonstimulated spines showed no changes in spine size (size variations, $1 \pm 3 \% ; n=10$ ). We then analyzed the distribution of EGFP-PAK3 and DsRedExpress by measuring the intensity of the green and red signals in the spine head and adjacent dendrite. To minimize possible sources of error, such as the variability in expression of fluorescent proteins and to compare images taken at different times, we systematically calculated green/red ratios and normalized them to the values obtained in the adjacent dendrite. We then compared these values before and $10 \mathrm{~min}$ after glutamate uncaging. A green/red ratio close to 1 for the spine head/adjacent dendrite values indicated an even distribution of EGFP-PAK3 with regard to DsRedExpress, whereas a ratio $>1$ suggested an accumulation of EGFP-PAK3 in spine heads as opposed to the adjacent dendrite. As shown in Figure $5 B--D$, light-stimulated spines enlarged and exhibited an increased EGFP-PAK3 fluorescence in their head. Calculations of green/red ratios before and 10-15 min after uncaging showed that this ratio did not vary under control conditions (light stimulation but no caged glutamate; light stimulation in EGFPtransfected cells; Fig. 5E). In contrast, in cells expressing EGFP$\mathrm{PAK} 3$, the green/red ratio significantly increased in lightstimulated spines but not in nonstimulated ones $(1.21 \pm 0.06$ vs $0.94 \pm 0.04 ; n=13$ and 9; $p<0.01$, Mann-Whitney $U$ test), indicating a selective recruitment of EGFP-PAK3 in activated spines. Additionally, we also measured green/red ratios in more distant part of the dendrite ( $>3 \mu \mathrm{m}$ apart) and normalized the values obtained in the adjacent dendrites to these more distal values before and after uncaging. The results show a decrease of adjacent versus distal measurements $10 \mathrm{~min}$ after versus before glutamate uncaging, an effect not seen in EGFP-transfected neurons $(0.92 \pm 0.02 \%, n=11$ vs $1.01 \pm 0.02 \%, n=7 ; p<0.05$, Mann-Whitney $U$ test). This effect is therefore consistent with the notion of a redistribution of PAK3-EGFP from the adjacent dendrites into activated spines. 
A
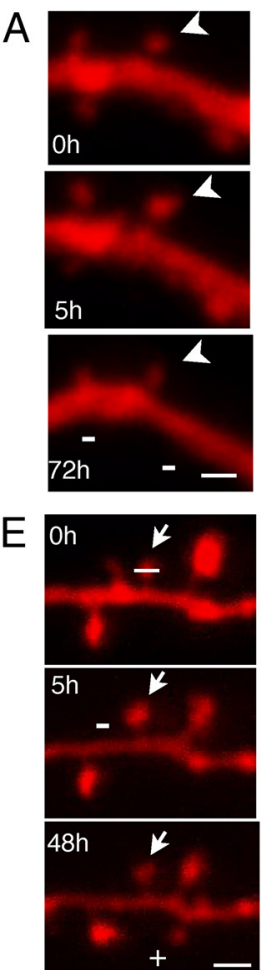

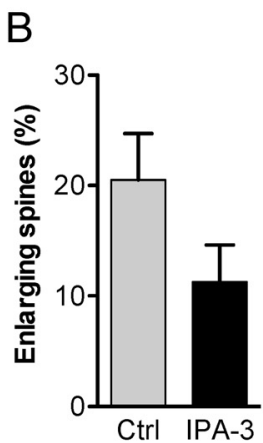

C
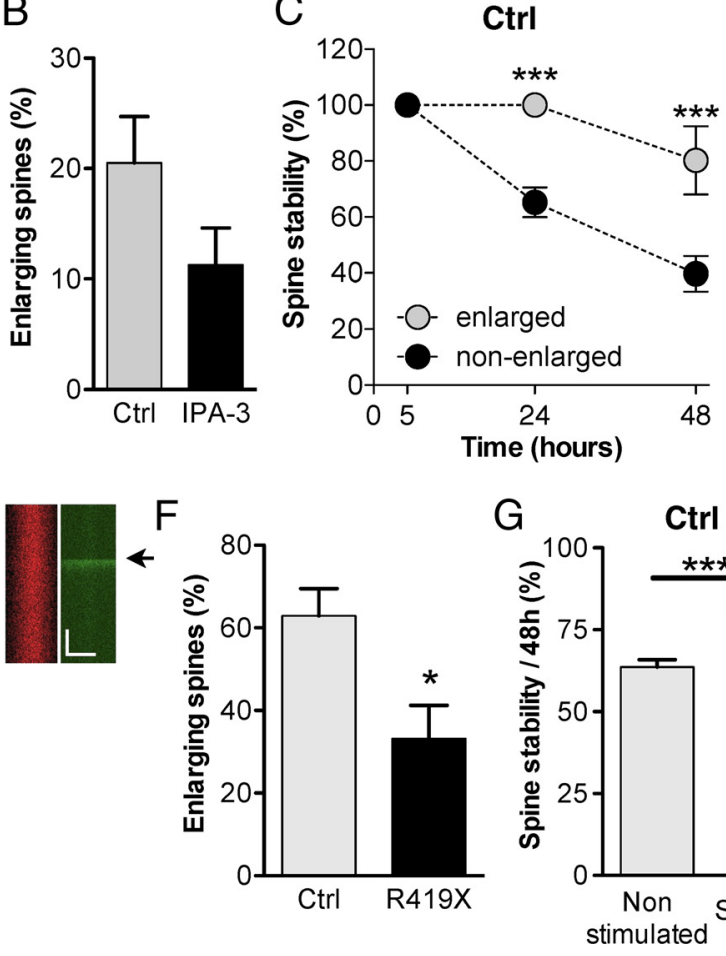
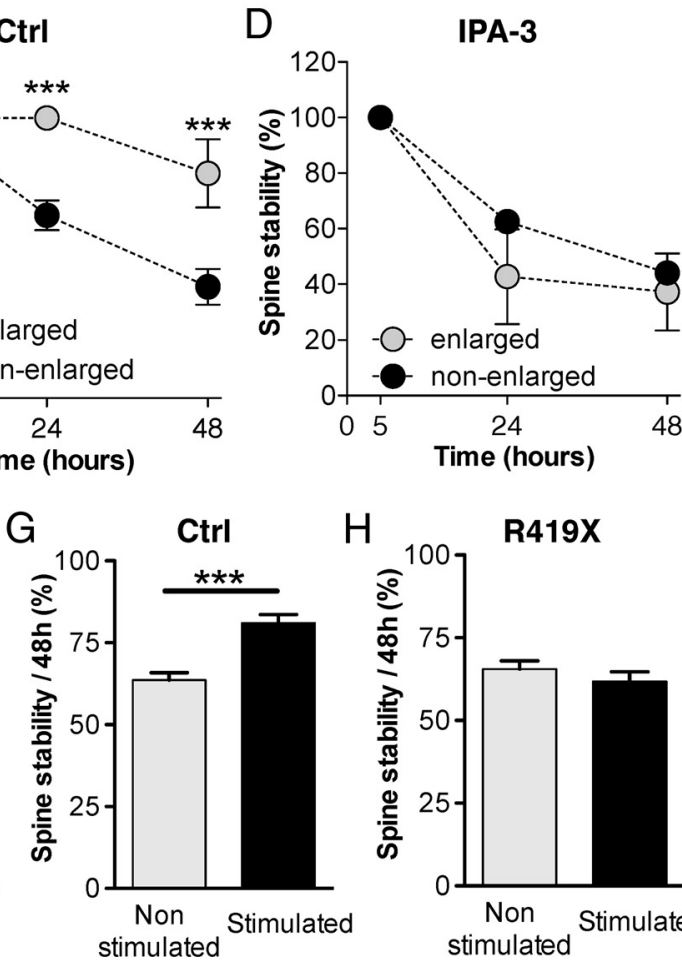

$\mathrm{H} \quad \mathbf{R 4 1 9 X}$

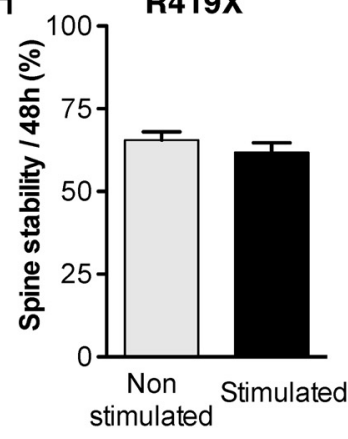

Figure 6. Inhibition of PAK3 prevents activity-mediated long-term stabilization of stimulated spines. $A$, TBS of slice cultures results in a transient enlargement of a fraction of spines that become more stable (arrowhead; scale bar, $1 \mu \mathrm{m}$ ). $\boldsymbol{B}$, The fraction of spines enlarging as a result of TBS tended to be lower after treatment with IPA-3 (15 $\mu \mathrm{m} ; n=4 ; p=0.1)$, The long-term stability of spines that enlarged $5 \mathrm{~h}$ after TBS (gray circles; $n=4$ cell, 27 spines analyzed) is increased compared with non-enlarged spines (black circles; $n=4$ cells, 111 spines analyzed; $p<0.001$, two-way ANOVA). $D$, In contrast, the same stimulation protocol applied to IPA-3-treated slices failed to enhance the stability of enlarged (gray circles; $n=$ 4 cells, 23 spines analyzed) versus non-enlarged spines (black circles; $n=4$ cells, 153 spines analyzed). $\boldsymbol{E}$, Dendritic segment of an mRFP-transfected neuron loaded with Fluo-4 AM and imaged at different times after TBS (scale bar, $2 \mu \mathrm{m}$ ). Line scans through the indicated spine (arrow) show an increase in fluorescence intensity of the green calcium signal at the time of stimulation (right panel, black arrow; calibration: $0.5 \mu \mathrm{m}, 0.5 \mathrm{~s}$ ). $\boldsymbol{F}$, The fraction of stimulated spines that showed an enlargement $5 \mathrm{~h}$ after TBS was decreased in R419X-PAK3transfected cells versus mRFP neurons ( $n=8$ cells, 81 and 64 spines analyzed; $p<0.05$ ). $\mathbf{G}$, Under control conditions (mRFP-transfected cells), the $48 \mathrm{~h}$ stability of stimulated spines was significantly enhanced versus nonstimulated spines ( $n=8$ cells, 37 and 44 spines analyzed; $p<0.001)$. $\boldsymbol{H}$, In contrast, the stability of stimulated spines in R419X-PAK3-transfected cells was no longer enhanced by stimulation ( $n=8$ cells, 30 and 34 spines analyzed). ${ }^{*} p<0.05 ;{ }^{* * *} p<0.001$.

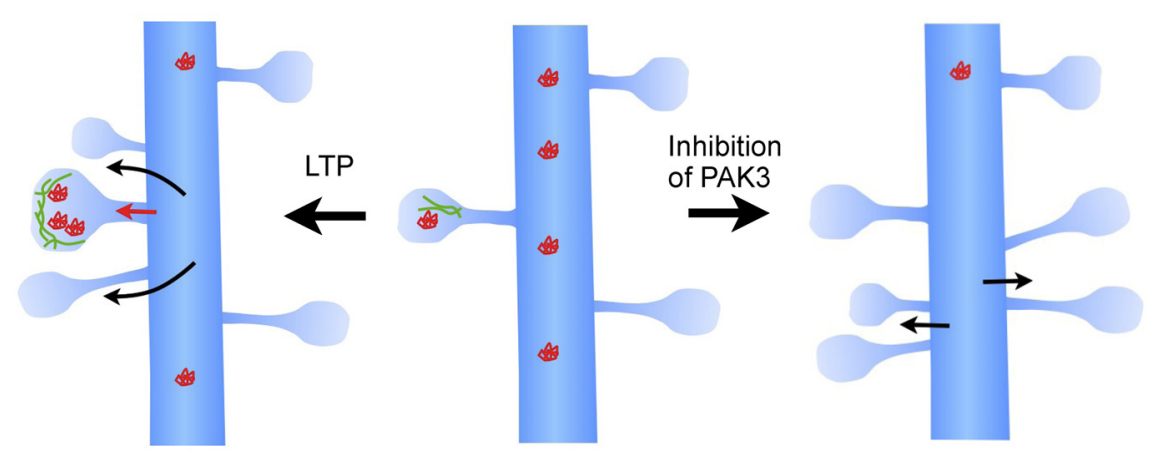

Figure 7. Schematic diagram illustrating the putative role of PAK3 in activity-mediated spine dynamics. Under control conditions, high-frequency stimulation of a spine (left) induces the recruitment of PAK3 into the spine head in which it contributes to the regulation of spine size and the long-term stabilization of the spine. Simultaneously, the redistribution of PAK3 results in a decrease of PAK3 in neighboring dendrites, thus relieving the inhibitory control of PAK3, leading to the local formation of spines. Under conditions of loss of PAK3 function (right), the inhibitory control of PAK3 on spine formation is reduced, leading to an excess of spine growth triggered by spontaneous or local activity.

\section{PAK3 contributes to an activity-dependent stabilization of spines}

To examine the role of PAK3 in activated spines, we tested the possibility that PAK3 could contribute to stabilize activated synapses. For this, we assessed the stability of spines over several days after induction of LTP using TBS. TBS resulted in the enlargement of a fraction of spines (Fig. $6 A, B$ ), and the spines that were enlarged (increase in spine head diameter $>0.1 \mu \mathrm{m}) 5 \mathrm{~h}$ after LTP stimulation exhibited a much higher stability over the next $48 \mathrm{~h}$ than the spines on the same dendrite that did not enlarge $(80 \pm 12$ vs $39 \pm$ $6 \%, n=4 ; p<0.001$, two-way ANOVA with Bonferroni's post hoc test; Fig. 6C). Replication of this experiment in the presence of $15 \mu \mathrm{M}$ IPA-3 revealed that the proportion of spines showing a substantial enlargement $(>0.1 \mu \mathrm{m})$ was reduced (Fig. 6B) and, more importantly, that the selective stabilizing effect was lost, because the stability of both enlarged and non-enlarged spines remained comparable $(37 \pm 13$ and $44 \pm 3 \%, n=4$; Fig. $6 D)$. Thus, LTP induction in the presence of IPA-3 failed to switch stimulated synapses to a stable state.

To confirm this in a more specific manner, we loaded R419X-PAK3-transfected CA1 neurons with a cell-permeable calcium indicator (Fluo-4 AM) using bulk loading. Stimulation of a group of CA3 neurons allowed in this way to identify spines on the same segment that showed or not a calcium signal in response to electrical stimulation (Fig. 6E). TBS was then applied using the same stimulation intensity to induce LTP, 
and we monitored the behavior of the spines that did or did not respond to stimulation over the next days. As indicated in Figure $6 F$, a majority of stimulated spines showed an enlargement of $>0.1 \mu \mathrm{m}$ of their spine head diameter $5 \mathrm{~h}$ later. Furthermore, and consistent with previous work (De Roo et al., 2008), LTP induction led to a selective long-term stabilization of stimulated synapses with a higher proportion of stimulated synapses still present $48 \mathrm{~h}$ later when compared with nonstimulated spines $(81.1 \pm 2.5$ vs $63 \pm 2.2 \%, n=37$ and 44 spines analyzed, $p<$ 0.001 ; Fig. $6 G$ ). In contrast, when the same procedure was applied to R419X-PAK3- and mRFP-cotransfected cells, the proportion of stimulated spines that enlarged was significantly decreased (33.1 \pm 8.0 vs $62.9 \pm 6.6 \%, n=7$ and 8 cells analyzed, $p<0.05$; Fig. $6 F$ ), and the activity-mediated stabilization effect was completely lost $(61.8 \pm 2.9$ vs $66.6 \pm 2.2 \%$ of spines still present at $48 \mathrm{~h}, n=30$ and 34 spines analyzed; Fig. $6 \mathrm{H}$ ). This result thus confirms that PAK3 function is required for plasticity to switch synapses to a stable state.

\section{Discussion}

This study reveals a novel function of the intellectual disability protein $\mathrm{PAK} 3$ in regulating the mechanisms of activitydependent reorganization of synaptic networks. Despite strong homologies between the different members of group I PAK family, the use of multiple approaches to target PAK3 strongly suggests that the effects reported here are attributable to PAK3. They are consistent with a model in which PAK3 plays a dual role in regulating two major aspects of activity-dependent and learningassociated structural plasticity: the growth of new protrusions and the stabilization of activated synapses (Fig. 7). Several recent studies showed that activity and learning behaviors are associated with an increased growth and turnover of spines as well as a selective stabilization of activated or new synapses (Holtmaat et al., 2006; De Roo et al., 2008; Hofer et al., 2009; Holtmaat and Svoboda, 2009; Xu et al., 2009; Yang et al., 2009; Roberts et al., 2010). These mechanisms are critical to allow adaptation and fine-tuning of synaptic networks by experience.

An important new finding of this study is the important role played by PAK3 in these mechanisms as a negative regulator of spine growth. This effect is activity dependent and suggests that PAK 3 could participate to a homeostatic control of spine number by activity. When mutated, the loss of PAK3 function enables an uncontrolled growth process, resulting in an excessive, nonspecific remodeling of brain circuits. Interestingly, this role of PAK3 could also account for the local spine growth associated with LTP induction (De Roo et al., 2008). Our data show that PAK3 is specifically recruited in the spine head of activated spines. Additionally, we find a small reduction of PAK3 in the nearby dendrite as opposed to more distal parts of the dendrite. This result therefore suggests that the redistribution of PAK3 relieves its negative action on spine growth in the nearby dendrite and thereby promotes a local formation of new spines, as seen with PAK3 inhibition. Under physiological conditions, PAK3 could therefore contribute to a local regulation by activity of spine formation, promoting the formation of new synapses in areas in which active partners can be found.

PAK3 is not the only molecule recruited by activity into spines. Profilin, another regulator of actin polymerization, is also targeted to spines heads in an NMDA receptor-dependent manner (Ackermann and Matus, 2003). There is also strong evidence for a redistribution of polyribosomes from dendritic shafts to active synapses (Ostroff et al., 2002). Activity might thus promote local movement of a number of molecules into spines, and PAK3 might not only be part of these changes but also participate in their regulation, notably through its possible action on proteins, such as cofilin, myosin II regulatory light chain, or cortactin (Edwards et al., 1999; Hashimoto et al., 2001; Zhang et al., 2005).

A second important finding of the study is the role of PAK3 in dendritic spine stabilization. PAKs are activated and phosphorylated in spines by stimulation patterns that induce LTP (Chen et al., 2007; Rex et al., 2009). This is associated with a structural remodeling of the spine and a transient enlargement of the spine head (Matsuzaki et al., 2004; De Roo et al., 2008; Yasumatsu et al., 2008; Xu et al., 2009). Rho GTPases and notably Cdc42 play an important role in controlling this step (Murakoshi et al., 2011), and our results, not surprisingly, indicate that PAK3 is also implicated in this effect. More importantly, however, we find that PAK3 inhibition prevented the switch of these stimulated spines to a stable state. Spine stabilization is likely to involve mechanisms that are probably related but distinct from the changes in size produced by induction of plasticity. Previous work has indeed shown that the stabilization is maintained, whereas the changes in size seem to be reversible and to fluctuate (De Roo et al., 2008; Yasumatsu et al., 2008). Spine stabilization is a key mechanism contributing to memory processes (De Roo et al., 2008; Xu et al., 2009; Yang et al., 2009), and the mechanisms underlying this property are still unclear. A recent study identified $\beta$-adducin, another cytoskeletal protein regulating $\mathrm{F}$-actin, as an important partner in this stabilization process (Bednarek and Caroni, 2011). Additionally, previous work showed that $\mathrm{N}$-cadherin, which may also signal through Rho GTPases, is implicated in long-term spine stabilization (Mendez et al., 2010). These results suggest that the regulation of the cytoskeleton, notably by Rho GTPase signaling, could play a major role in the control of spine stability.

Together, this study indicates that PAK3 participates to the regulation of two essential mechanisms for the development of synaptic networks. Loss of PAK3 function, such as in patients with PAK3 mutation, leads to an uncontrolled growth of new spines that introduce nonspecificity in the wiring of developing networks and an impairment in activity-mediated spine stabilization, affecting the capacity to maintain important connections. The alterations associated with PAK3 mutation might thus share analogies with those reported in studies of fragile $\mathrm{X}$ knock-out mice (Cruz-Martín et al., 2010; Pan et al., 2010). These alterations are likely to affect the organization and connectivity of brain circuits and provide additional evidence that defects in synapse dynamics during development could represent an important mechanism to account for intellectual disability.

\section{References}

Ackermann M, Matus A (2003) Activity-induced targeting of profilin and stabilization of dendritic spine morphology. Nat Neurosci 6:1194-1200.

Allen KM, Gleeson JG, Bagrodia S, Partington MW, MacMillan JC, Cerione RA, Mulley JC, Walsh CA (1998) PAK3 mutation in nonsyndromic X-linked mental retardation. Nat Genet 20:25-30.

Bednarek E, Caroni P (2011) beta-Adducin is required for stable assembly of new synapses and improved memory upon environmental enrichment. Neuron 69:1132-1146.

Boda B, Alberi S, Nikonenko I, Node-Langlois R, Jourdain P, Moosmayer M, Parisi-Jourdain L, Muller D (2004) The mental retardation protein PAK3 contributes to synapse formation and plasticity in hippocampus. J Neurosci 24:10816-10825.

Chen LY, Rex CS, Casale MS, Gall CM, Lynch G (2007) Changes in synaptic morphology accompany actin signaling during LTP. J Neurosci 27: 5363-5372. 
Cruz-Martín A, Crespo M, Portera-Cailliau C (2010) Delayed stabilization of dendritic spines in fragile X mice. J Neurosci 30:7793-7803.

Dawson PE, Muir TW, Clark-Lewis I, Kent SB (1994) Synthesis of proteins by native chemical ligation. Science 266:776-779.

De Roo M, Klauser P, Muller D (2008) LTP promotes a selective long-term stabilization and clustering of dendritic spines. PLoS Biol 6:e219.

Edwards DC, Sanders LC, Bokoch GM, Gill GN (1999) Activation of LIMkinase by Pak1 couples Rac/Cdc42 GTPase signalling to actin cytoskeletal dynamics. Nat Cell Biol 1:253-259.

Harvey CD, Svoboda K (2007) Locally dynamic synaptic learning rules in pyramidal neuron dendrites. Nature 450:1195-1200.

Hashimoto S, Tsubouchi A, Mazaki Y, Sabe H (2001) Interaction of paxillin with p21-activated Kinase (PAK). Association of paxillin alpha with the kinase-inactive and the Cdc42-activated forms of PAK3. J Biol Chem 276:6037-6045.

Hayashi-Takagi A, Takaki M, Graziane N, Seshadri S, Murdoch H, Dunlop AJ, Makino Y, Seshadri AJ, Ishizuka K, Srivastava DP, Xie Z, Baraban JM, Houslay MD, Tomoda T, Brandon NJ, Kamiya A, Yan Z, Penzes P, Sawa A (2010) Disrupted-in-Schizophrenia 1 (DISC1) regulates spines of the glutamate synapse via Rac1. Nat Neurosci 13:327-332.

Hofer SB, Mrsic-Flogel TD, Bonhoeffer T, Hübener M (2009) Experience leaves a lasting structural trace in cortical circuits. Nature 457:313-317.

Holtmaat A, Svoboda K (2009) Experience-dependent structural synaptic plasticity in the mammalian brain. Nat Rev Neurosci 10:647-658.

Holtmaat A, Wilbrecht L, Knott GW, Welker E, Svoboda K (2006) Experience-dependent and cell-type-specific spine growth in the neocortex. Nature 441:979-983.

Kreis P, Thévenot E, Rousseau V, Boda B, Muller D, Barnier JV (2007) The p21-activated kinase 3 implicated in mental retardation regulates spine morphogenesis through a Cdc42-dependent pathway. J Biol Chem 282:21497-21506.

Lüthi A, Schwyzer L, Mateos JM, Gähwiler BH, McKinney RA (2001) NMDA receptor activation limits the number of synaptic connections during hippocampal development. Nat Neurosci 4:1102-1107.

Mameli M, Bellone C, Brown MT, Lüscher C (2011) Cocaine inverts rules for synaptic plasticity of glutamate transmission in the ventral tegmental area. Nat Neurosci 14:414-416.

Mateos JM, Lüthi A, Savic N, Stierli B, Streit P, Gähwiler BH, McKinney RA (2007) Synaptic modifications at the CA3-CA1 synapse after chronic AMPA receptor blockade in rat hippocampal slices. J Physiol 581: $129-138$.

Matsuzaki M, Honkura N, Ellis-Davies GC, Kasai H (2004) Structural basis of long-term potentiation in single dendritic spines. Nature 429:761-766.

Mendez P, De Roo M, Poglia L, Klauser P, Muller D (2010) N-cadherin mediates plasticity-induced long-term spine stabilization. J Cell Biol 189:589-600

Meng J, Meng Y, Hanna A, Janus C, Jia Z (2005) Abnormal long-lasting synaptic plasticity and cognition in mice lacking the mental retardation gene Pak3. J Neurosci 25:6641-6650.

Murakoshi H, Wang H, Yasuda R (2011) Local, persistent activation of Rho GTPases during plasticity of single dendritic spines. Nature 472:100-104.

Ostroff LE, Fiala JC, Allwardt B, Harris KM (2002) Polyribosomes redistribute from dendritic shafts into spines with enlarged synapses during LTP in developing rat hippocampal slices. Neuron 35:535-545.

Pan F, Aldridge GM, Greenough WT, Gan WB (2010) Dendritic spine instability and insensitivity to modulation by sensory experience in a mouse model of fragile X syndrome. Proc Natl Acad Sci U S A 107:17768-17773.

Penzes P, Cahill ME, Jones KA, VanLeeuwen JE, Woolfrey KM (2011) Dendritic spine pathology in neuropsychiatric disorders. Nat Neurosci 14:285-293.

Rex CS, Chen LY, Sharma A, Liu J, Babayan AH, Gall CM, Lynch G (2009) Different Rho GTPase-dependent signaling pathways initiate sequential steps in the consolidation of long-term potentiation. J Cell Biol 186:85-97.

Roberts TF, Tschida KA, Klein ME, Mooney R (2010) Rapid spine stabilization and synaptic enhancement at the onset of behavioural learning. $\mathrm{Na}$ ture 463:948-952.

Ropers HH (2008) Genetics of intellectual disability. Curr Opin Genet Dev $18: 241-250$

Stoppini L, Buchs PA, Muller D (1991) A simple method for organotypic cultures of nervous tissue. J Neurosci Methods 37:173-182.

Wilbrecht L, Holtmaat A, Wright N, Fox K, Svoboda K (2010) Structural plasticity underlies experience-dependent functional plasticity of cortical circuits. J Neurosci 30:4927-4932.

Xie Z, Srivastava DP, Photowala H, Kai L, Cahill ME, Woolfrey KM, Shum CY, Surmeier DJ, Penzes P (2007) Kalirin-7 controls activity-dependent structural and functional plasticity of dendritic spines. Neuron 56:640 656.

Xu T, Yu X, Perlik AJ, Tobin WF, Zweig JA, Tennant K, Jones T, Zuo Y (2009) Rapid formation and selective stabilization of synapses for enduring motor memories. Nature 462:915-919.

Yang G, Pan F, Gan WB (2009) Stably maintained dendritic spines are associated with lifelong memories. Nature 462:920-924.

Yasumatsu N, Matsuzaki M, Miyazaki T, Noguchi J, Kasai H (2008) Principles of long-term dynamics of dendritic spines. J Neurosci 28:1359213608.

Zhang H, Webb DJ, Asmussen H, Niu S, Horwitz AF (2005) A GIT1/PIX/ Rac/PAK signaling module regulates spine morphogenesis and synapse formation through MLC. J Neurosci 25:3379-3388. 